Pacific

Journal of

Mathematics

\title{
STRUCTURE OF SEEDS IN GENERALIZED
} CLUSTER ALGEBRAS

TOMOKI NAKANISHI

Volume $277 \quad$ No. 1

September 2015 


\title{
STRUCTURE OF SEEDS IN GENERALIZED CLUSTER ALGEBRAS
}

\author{
TOMOKI NAKANISHI
}

\begin{abstract}
We study generalized cluster algebras, introduced by Chekhov and Shapiro. When the coefficients satisfy the normalization and quasireciprocity conditions, one can naturally extend the structure theory of seeds in the ordinary cluster algebras by Fomin and Zelevinsky to generalized cluster algebras. As the main result, we obtain formulas expressing cluster variables and coefficients in terms of $c$-vectors, $g$-vectors, and $\boldsymbol{F}$-polynomials.
\end{abstract}

\section{Introduction}

Chekhov and Shapiro [2014] introduced generalized cluster algebras, which naturally generalize the ordinary cluster algebras by Fomin and Zelevinsky [2002]. In generalized cluster algebras, the celebrated binomial exchange relation for cluster variables of ordinary cluster algebras

$$
\begin{aligned}
x_{k}^{\prime} x_{k} & =p_{k}^{-} \prod_{j=1}^{n} x_{j}^{\left[-b_{j k}\right]_{+}}+p_{k}^{+} \prod_{j=1}^{n} x_{j}^{\left[b_{j k}\right]_{+}} \\
& =\left(\prod_{j=1}^{n} x_{j}^{\left[-b_{j k}\right]_{+}}\right)\left(p_{k}^{-}+p_{k}^{+} w_{k}\right), \quad w_{k}=\prod_{j=1}^{n} x_{j}^{b_{j k}},
\end{aligned}
$$

is replaced by the polynomial one of arbitrary degree $d_{k} \geq 1$,

$$
x_{k}^{\prime} x_{k}=\left(\prod_{j=1}^{n} x_{j}^{\left[-\beta_{j k}\right]_{+}}\right)^{d_{k}} \sum_{s=0}^{d_{k}} p_{k, s} w_{k}^{s}, \quad w_{k}=\prod_{j=1}^{n} x_{j}^{\beta_{j k}},
$$

where $\beta_{j k}=b_{j k} / d_{k}$ are assumed to be integers and the coefficients $p_{k, s}$ should also be mutated appropriately. This generalization is expected to be natural, since it originates in the transformations preserving the associated Poisson bracket [Gekhtman et al. 2005]. In fact, it was shown in [Chekhov and Shapiro 2014] that the generalized cluster algebras have the Laurent property, which is regarded as the most characteristic feature of the ordinary cluster algebras. It was also shown in

MSC2010: 13F60.

Keywords: cluster algebra. 
the same paper that the finite-type classification of the generalized cluster algebras reduces to the one for the ordinary case. These results already imply that, despite the apparent complexity of their exchange relations (1-2), generalized cluster algebras may be well controlled like the ordinary ones. See also [Rupel 2013] for the result on greedy bases in rank 2 generalized cluster algebras.

Besides the above cluster-algebra-theoretic interest, the generalized cluster algebra structure naturally appears for the Teichmüller spaces of Riemann surfaces with orbifold points [Chekhov and Shapiro 2014]. More recently, it also appears in representation theory of quantum affine algebras [Gleitz 2014] and also in the study of WKB analysis [Iwaki and Nakanishi 2014]. In view of these developments, and also for potentially more versatility of polynomial exchange relations than the binomial one, it is not only natural but also necessary to develop a structure theory of seeds in generalized cluster algebras which is parallel to the one for the ordinary cluster algebras by [Fomin and Zelevinsky 2007]. The core notion of the theory of that paper is a cluster pattern with principal coefficients, from which other important notions such as $c$-vectors, $g$-vectors, and $F$-polynomials are also induced. Then, the main result of [Fomin and Zelevinsky 2007] is the formulas expressing cluster variables and coefficients in terms of $c$-vectors, $g$-vectors, and $F$-polynomials. These formulas are especially important in view of the categorification of cluster algebras by (generalized) cluster categories (see [Plamondon 2011] and references therein).

The purpose of this paper is to provide results parallel to the above ones for generalized cluster algebras. To be more precise, we consider a class of generalized cluster algebras whose coefficients satisfy the normalization condition and what we call the quasireciprocity condition. For this class of generalized cluster algebras, we introduce the notions of a cluster pattern with principal coefficients, $c$-vectors, $g$-vectors, and $F$-polynomials. Then, as a main result, we obtain the formulas expressing cluster variables and coefficients in terms of $c$-vectors, $g$-vectors, and $F$-polynomials, which are parallel to the ones in [Fomin and Zelevinsky 2007]. To summarize, generalized cluster algebras preserve essentially every feature of the ordinary ones, and this is the main message of the paper.

\section{Generalized cluster algebras}

In this section we recall basic notions of generalized cluster algebras following [Chekhov and Shapiro 2014]. However, we slightly modify the setting of Chekhov and Shapiro to match the setting of (ordinary) cluster algebras in [Fomin and Zelevinsky 2007].

2A. Generalized seed mutations. Throughout the paper we always assume that any matrix is an integer matrix. 
Recall that a matrix $B=\left(b_{i j}\right)_{i . j=1}^{n}$ is said to be skew-symmetrizable if there is an $n$-tuple of positive integers $\boldsymbol{d}=\left(d_{1}, \ldots, d_{n}\right)$ such that $d_{i} b_{i j}=-d_{j} b_{j i}$.

We start by fixing a semifield $\mathbb{P}$, whose addition is denoted by $\oplus$. Let $\mathbb{Z} \mathbb{P}$ be the group ring of $\mathbb{P}$, and let $\mathbb{Q P}$ be the field of fractions of $\mathbb{Z} \mathbb{P}$. Let $w_{1}, \ldots, w_{n}$ be any algebraic independent variables, and let $\mathcal{F}=\mathbb{Q P}(w)$ be the field of rational functions in $w=\left(w_{1}, \ldots, w_{n}\right)$ with coefficients in $\mathbb{Q P P}$.

The following definition is the usual one [Fomin and Zelevinsky 2007].

Definition 2.1. A (labeled) seed in $\mathbb{P}$ is a triplet $(\boldsymbol{x}, \boldsymbol{y}, B)$ such that

- $B$ is a skew-symmetrizable matrix, called an exchange matrix,

- $\boldsymbol{x}=\left(x_{1}, \ldots, x_{n}\right)$ is an $n$-tuple of elements in $\mathcal{F}$, called cluster variables or $x$-variables,

- $\boldsymbol{y}=\left(y_{1}, \ldots, y_{n}\right)$ is an $n$-tuple of elements in $\mathbb{P}$, called coefficients or $y$ variables.

Next we introduce a pair $(\boldsymbol{d}, \boldsymbol{z})$ of data for generalized seed mutations. Firstly, $\boldsymbol{d}=\left(d_{1}, \ldots, d_{n}\right)$ is an $n$-tuple of positive integers, and we call these integers the mutation degrees. We stress that we do not impose the skew-symmetric condition $d_{i} b_{i j}=-d_{j} b_{j i}$. Secondly, $z$ is a family of elements in $\mathbb{P}$,

$$
z=\left(z_{i, s}\right)_{i=1, \ldots, n ; s=1, \ldots, d_{i}-1},
$$

satisfying the reciprocity condition

$$
z_{i, s}=z_{i, d_{i}-s} \quad\left(s=1, \ldots, d_{i}-1\right) .
$$

We call them the frozen coefficients, since they are not "mutated", or simply the $z$-variables. We also set

$$
z_{i, 0}=z_{i, d_{i}}=1 .
$$

For $\boldsymbol{d}=(1, \ldots, 1), \boldsymbol{z}$ is empty, and it reduces to the ordinary case. (Here and below, "ordinary" means the case of ordinary cluster algebras.)

Definition 2.2. Let $(\boldsymbol{d}, \boldsymbol{z})$ be given as above. For any seed $(\boldsymbol{x}, \boldsymbol{y}, B)$ in $\mathbb{P}$ and $k=1, \ldots, n$, the $(\boldsymbol{d}, \boldsymbol{z})$-mutation of $(\boldsymbol{x}, \boldsymbol{y}, B)$ at $k$ is another seed $\left(\boldsymbol{x}^{\prime}, \boldsymbol{y}^{\prime}, B^{\prime}\right)=$ $\mu_{k}(\boldsymbol{x}, \boldsymbol{y}, B)$ in $\mathbb{P}$ defined by the following rule:

$$
\begin{gathered}
b_{i j}^{\prime}= \begin{cases}-b_{i j} & \text { if } i=k \text { or } j=k, \\
b_{i j}+d_{k}\left(\left[-b_{i k}\right]_{+} b_{k j}+b_{i k}\left[b_{k j}\right]_{+}\right) & \text {if } i, j \neq k,\end{cases} \\
y_{i}^{\prime}= \begin{cases}y_{k}^{-1} & \text { if } i=k, \\
y_{i}\left(y_{k}^{\left[\varepsilon b_{k i}\right]_{+}}\right)^{d_{k}}\left(\bigoplus_{s=0}^{d_{k}} z_{k, s} y_{k}^{\varepsilon s}\right)^{-b_{k i}} & \text { if } i \neq k,\end{cases}
\end{gathered}
$$




$$
x_{i}^{\prime}= \begin{cases}x_{k}^{-1}\left(\prod_{j=1}^{n} x_{j}^{\left[-\varepsilon b_{j k}\right]_{+}}\right)^{d_{k}} \frac{\sum_{s=0}^{d_{k}} z_{k, s} \hat{y}_{k}^{\varepsilon s}}{\bigoplus_{k} z_{k, s} y_{k}^{\varepsilon s}} & \text { if } i=k, \\ x_{i} & \text { if } i \neq k,\end{cases}
$$

where $\varepsilon= \pm 1,[a]_{+}=\max (a, 0)$, and we set

$$
\hat{y}_{i}=y_{i} \prod_{j=1}^{n} x_{j}^{b_{j i}} .
$$

When the data $(\boldsymbol{d}, \boldsymbol{z})$ is clearly assumed, we may drop the prefix and simply call it the (generalized) mutation.

Let $D=\left(d_{i} \delta_{i j}\right)_{i, j=1}^{n}$ be the diagonal matrix with diagonal entries $\boldsymbol{d}$. It is important to note that the mutation (2-4) is equivalent to the ordinary mutation of exchange matrices between $D B$ and $D B^{\prime}$, and also between $B D$ and $B^{\prime} D$ in [Fomin and Zelevinsky 2007].

The following properties are easy to confirm:

- The formulas (2-5) and (2-6) are independent of the choice of the sign $\varepsilon$ due to $(2-2)$.

- The mutation $\mu_{k}$ is involutive, i.e., $\mu_{k}\left(\mu_{k}(\boldsymbol{x}, \boldsymbol{y}, B)\right)=(\boldsymbol{x}, \boldsymbol{y}, B)$.

Remark 2.3. Here we transposed every matrix in [Chekhov and Shapiro 2014]. Also, the matrix $B$ therein is the matrix $D B^{T}$ here, and $\beta_{i j}$ therein is $b_{j i}$ here.

Remark 2.4. In this paper we do not use the freedom of the choice of sign $\varepsilon$ in (2-5) and (2-6), and it can be safely set as $\varepsilon=1$ throughout. Nevertheless, we keep it in all formulas involved since it is useful for several purposes, for example, to consider signed mutations, which appeared in [Iwaki and Nakanishi 2014].

Proposition 2.5. Under the mutation $\mu_{k}$, the $\hat{y}$-variables (2-7) mutate in the same way as the $y$-variables, namely,

$$
\hat{y}_{i}^{\prime}= \begin{cases}\hat{y}_{k}^{-1} & \text { if } i=k, \\ \hat{y}_{i}\left(\hat{y}_{k}^{\left[\varepsilon b_{k i}\right]_{+}}\right)^{d_{k}}\left(\sum_{s=0}^{d_{k}} z_{k, s} \hat{y}_{k}^{\varepsilon s}\right)^{-b_{k i}} & \text { if } i \neq k .\end{cases}
$$

Proof. This is proved using the technique in [Fomin and Zelevinsky 2007, Proposition 3.9].

Next let us explain how our setting is regarded as a specialization of the setting of [Chekhov and Shapiro 2014]. In that paper a seed in $\mathbb{P}$ is defined as a triplet 
$(\boldsymbol{x}, \boldsymbol{p}, B)$, where $\boldsymbol{x}$ and $B$ are the same as in this paper (up to the identification of $B$ as in Remark 2.3 ), but $\boldsymbol{p}$ is a family of elements in $\mathbb{P}$,

$$
\boldsymbol{p}=\left(p_{i, s}\right)_{i=1, \ldots, n ; s=0, \ldots, d_{i}} .
$$

Then, for the mutation $\left(\boldsymbol{x}^{\prime}, \boldsymbol{p}^{\prime}, B^{\prime}\right)=\mu_{k}(\boldsymbol{x}, \boldsymbol{p}, B)$, the following formulas replace (2-5) and (2-6):

$$
\begin{aligned}
p_{k, s}^{\prime} & =p_{k, d_{k}-s}, \\
\frac{p_{i, s}^{\prime}}{p_{i, 0}^{\prime}} & = \begin{cases}\frac{p_{i, s}}{p_{i, 0}}\left(p_{k, d_{k}}^{b_{k i}}\right)^{s} & \text { if } i \neq k, b_{k i} \geq 0, \\
\frac{p_{i, s}}{p_{i, 0}}\left(p_{k, 0}^{b_{k i}}\right)^{s} & \text { if } i \neq k, b_{k i} \leq 0,\end{cases} \\
x_{i}^{\prime} & = \begin{cases}x_{k}^{-1}\left(\prod_{j=1}^{n} x_{j}^{\left[-b_{j k}\right]_{+}}\right)^{d_{k}}\left(\sum_{s=0}^{d_{k}} p_{k, s} u_{k}^{s}\right) & \text { if } i=k, \\
x_{i} & \text { if } i \neq k,\end{cases}
\end{aligned}
$$

where

$$
u_{i}=\prod_{j=1}^{n} x_{j}^{b_{j i}} .
$$

Now, let us start from a seed $(\boldsymbol{x}, \boldsymbol{y}, B)$ in our setting. Comparing (2-6) and (2-11), we naturally identify

$$
p_{i, s}=\frac{z_{i, s} y_{i}^{s}}{\bigoplus_{r=0}^{d_{i}} z_{i, r} y_{i}^{r}} .
$$

Then, it is easy to check that the mutation (2-10) follows from (2-2) and (2-5). Moreover, the specialization (2-13) satisfies the normalization property

$$
\bigoplus_{s=0}^{d_{i}} p_{i, s}=1
$$

and the quasireciprocity property that for each $i=1, \ldots, n$ there is some $y_{i} \in \mathbb{P}$ such that

$$
\frac{p_{i, s}}{p_{i, 0}} \frac{p_{i, d_{i}}}{p_{i, d_{i}-s}}=y_{i}^{2 s}, \quad s=1, \ldots, d_{i} .
$$

Conversely, suppose that a family $\boldsymbol{p}$ in (2-9) satisfies properties (2-14) and (2-15). First we note that such a $y_{i}$ is unique, since any semifield $\mathbb{P}$ is torsion-free [Fomin and Zelevinsky 2002, Section 5]. Next we define $z_{i, s} \in \mathbb{P}(i=1, \ldots, n$; $s=0, \ldots, d_{i}$ ) by

$$
\frac{p_{i, s}}{p_{i, 0}}=y_{i}^{s} z_{i, s} .
$$


In particular, we have $z_{i, 0}=1$. Then, substituting (2-16) in (2-15), we obtain

$$
z_{i, s} z_{i, d_{i}} z_{i, d_{i}-s}^{-1}=1, \quad s=1, \ldots, d_{i} .
$$

In particular, by setting $s=d_{i}$, we have $z_{i, d_{i}}^{2}=1$. Once again, since $\mathbb{P}$ is torsion-free, we have $z_{i, d_{i}}=1$. Then, again by (2-17), we have the reciprocity $z_{i, s}=z_{i, d_{i}-s}$ $\left(s=1, \ldots, d_{i}-1\right)$. Meanwhile, by (2-14) and (2-16), we have

$$
p_{i, 0}=\frac{1}{\bigoplus_{s=0}^{d_{i}} z_{i, s} y_{i}^{s}} .
$$

Then, by (2-16) again, we recover the specialization (2-13). Finally, it is straightforward to recover the mutation (2-5) from (2-10) and (2-15). Furthermore, by (2-16), one can also confirm that the coefficients $z_{i, s}$ do not mutate.

2B. Generalized cluster algebras and Laurent property. Let $\mathbb{T}_{n}$ be the $n$-regular tree whose edges are labeled by the numbers $1, \ldots, n$. Following [Fomin and Zelevinsky 2002], let us write $t \underline{k} t^{\prime}$ if the vertices $t$ and $t^{\prime}$ of $\mathbb{T}_{n}$ are connected by the edge labeled by $k$.

Definition 2.6. A $(\boldsymbol{d}, \boldsymbol{z})$-cluster pattern $\Sigma$ in $\mathbb{P}$ is an assignment of a seed $\Sigma_{t}$ in $\mathbb{P}$ to each vertex $t$ of $\mathbb{T}$ such that if $t \underline{k} t^{\prime}$ then the assigned seeds $\Sigma_{t}$ and $\Sigma_{t^{\prime}}$ are obtained from each other by the $(\boldsymbol{d}, \boldsymbol{z})$-mutation at $k$.

We fix a vertex $t_{0}$ of $\mathbb{T}_{n}$ and call it the initial vertex. Accordingly, the assigned seed $\Sigma_{t_{0}}=\left(\boldsymbol{x}_{t_{0}}, \boldsymbol{y}_{t_{0}}, B_{t_{0}}\right)$ at $t_{0}$ is called the initial seed. Let us write, for simplicity,

$$
\boldsymbol{x}_{t_{0}}=\boldsymbol{x}=\left(x_{1}, \ldots, x_{n}\right), \quad \boldsymbol{y}_{t_{0}}=\boldsymbol{y}=\left(y_{1}, \ldots, y_{n}\right), \quad B_{t_{0}}=B=\left(b_{i j}\right)_{i, j=1}^{n} \text {. }
$$

On the other hand, for the seed $\Sigma_{t}=\left(\boldsymbol{x}_{t}, \boldsymbol{y}_{t}, B_{t}\right)$ assigned to a general vertex $t$ of $\mathbb{T}_{n}$, we write

$$
\boldsymbol{x}_{t}=\left(x_{1}^{t}, \ldots, x_{n}^{t}\right), \quad \boldsymbol{y}_{t}=\left(y_{1}^{t}, \ldots, y_{n}^{t}\right), \quad B_{t}=\left(b_{i j}^{t}\right)_{i, j=1}^{n} .
$$

Definition 2.7. The generalized cluster algebra $\mathcal{A}$ associated with a $(\boldsymbol{d}, \boldsymbol{z})$-cluster pattern $\Sigma$ in $\mathbb{P}$ is a $\mathbb{Z} \mathbb{P}$-subalgebra of $\mathcal{F}$ generated by all $x$-variables $x_{i}^{t}(t \in \mathbb{T}$, $i=1, \ldots, n)$ occurring in $\Sigma$. It is denoted by $\mathcal{A}=\mathcal{A}(\boldsymbol{x}, \boldsymbol{y}, B ; \boldsymbol{d}, \boldsymbol{z})$, where $(\boldsymbol{x}, \boldsymbol{y}, B)$ is the initial seed of $\Sigma$.

For any $(\boldsymbol{d}, \boldsymbol{z})$-cluster pattern in $\mathbb{P}$, each $x$-variable $x_{i}^{t}$ is expressed as a subtractionfree rational function of $\boldsymbol{x}$ with coefficients in $\mathbb{Q P}$. The following stronger property due to [Chekhov and Shapiro 2014] is of fundamental importance.

Theorem 2.8 (Laurent property [Chekhov and Shapiro 2014, Theorem 2.5]). For any $(\boldsymbol{d}, \boldsymbol{z})$-cluster pattern in $\mathbb{P}$, each $x$-variable $x_{i}^{t}$ is expressed as a Laurent polynomial of $\boldsymbol{x}$ with coefficients in $\mathbb{Z} \mathbb{P}$. 
2C. Example. As the simplest nontrivial example, we consider $\boldsymbol{d}=(2,1), \boldsymbol{z}=$ $\left(z_{1,1}\right)$, and an initial seed $(\boldsymbol{x}, \boldsymbol{y}, B)$ in $\mathbb{P}$ such that

$$
B=\left(\begin{array}{rr}
0 & -1 \\
1 & 0
\end{array}\right) .
$$

(This example also appears in [Chekhov and Shapiro 2014, proof of Theorem 2.7].) Accordingly,

$$
\hat{y}_{1}=y_{1} x_{2}, \quad \hat{y}_{2}=y_{2} x_{1}^{-1}
$$

We note that

$$
D B=\left(\begin{array}{rr}
0 & -2 \\
1 & 0
\end{array}\right), \quad B D=\left(\begin{array}{rr}
0 & -1 \\
2 & 0
\end{array}\right),
$$

which are the initial exchange matrices for ordinary cluster algebras of type $B_{2}=C_{2}$. Set $\Sigma(1)=(\boldsymbol{x}(1), \boldsymbol{y}(1), B(1))$ to be the initial seed $(\boldsymbol{x}, \boldsymbol{y}, B)$, and consider the seeds $\Sigma(t)=(\boldsymbol{x}(t), \boldsymbol{y}(t), B(t))(t=2, \ldots, 7)$ obtained by the following sequence of alternative mutations of $\mu_{1}$ and $\mu_{2}$.

$$
\Sigma(1) \stackrel{\mu_{1}}{\leftrightarrow} \Sigma(2) \stackrel{\mu_{2}}{\leftrightarrow} \Sigma(3) \stackrel{\mu_{1}}{\leftrightarrow} \Sigma(4) \stackrel{\mu_{2}}{\leftrightarrow} \Sigma(5) \stackrel{\mu_{1}}{\leftrightarrow} \Sigma(6) \stackrel{\mu_{2}}{\leftrightarrow} \Sigma(7) .
$$

By (2-4), we have

$$
B(t)=(-1)^{t+1} B .
$$

Then, using the exchange relations (2-5) and (2-6), we obtain the explicit expressions of $x$ - and $y$-variables in Table 1 , where we set $z_{1,1}=z$ for simplicity. We observe the same periodicity of mutations of seeds for the ordinary cluster algebras of type $B_{2}=C_{2}$.

\section{Structure of seeds in generalized cluster patterns}

The goal of this section is to establish some basic structural results on seeds in a $(\boldsymbol{d}, \boldsymbol{z})$-cluster pattern which are parallel to the ones in [Fomin and Zelevinsky 2007].

3A. $\boldsymbol{X}$-functions and $\boldsymbol{Y}$-functions. Let us temporarily regard $\boldsymbol{y}=\left(y_{i}\right)_{i=1}^{n}$ and $\boldsymbol{z}=\left(z_{i, s}\right)_{i=1, \ldots, n ; s=1, \ldots, d_{i}-1}$ with $z_{i, s}=z_{i, d_{i}-s}$ as formal variables. Let $\mathbb{Q}_{\mathrm{sf}}(\boldsymbol{y}, \boldsymbol{z})$ be the universal semifield of $\boldsymbol{y}$ and $\boldsymbol{z}$, which consists of the rational functions in $\boldsymbol{y}$ and $\boldsymbol{z}$ with subtraction-free expressions [Fomin and Zelevinsky 2007]. Let $\operatorname{Trop}(\boldsymbol{y}, \boldsymbol{z})$ be the tropical semifield of $\boldsymbol{y}$ and $\boldsymbol{z}$, which is the multiplicative abelian group freely generated by $\boldsymbol{y}$ and $\boldsymbol{z}$ with tropical sum $\oplus$ defined by

$$
\left(\prod_{i} y_{i}^{a_{i}} \prod_{i, s} z_{i, s}^{a_{i, s}}\right) \oplus\left(\prod_{i} y_{i}^{b_{i}} \prod_{i, s} z_{i, s}^{b_{i, s}}\right)=\prod_{i} y_{i}^{\min \left(a_{i}, b_{i}\right)} \prod_{i, s} z_{i, s}^{\min \left(a_{i, s}, b_{i, s}\right)} .
$$




$$
\begin{aligned}
& \left\{\begin{array}{l}
x_{1}(1)=x_{1} \\
x_{2}(1)=x_{2}
\end{array}\right. \\
& \left\{\begin{array}{l}
y_{1}(1)=y_{1} \\
y_{2}(1)=y_{2}
\end{array}\right. \\
& \left\{\begin{array}{l}
x_{1}(2)=x_{1}^{-1} \frac{1+z \hat{y}_{1}+\hat{y}_{1}^{2}}{1 \oplus z y_{1} \oplus y_{1}^{2}} \\
x_{2}(2)=x_{2}
\end{array}\right. \\
& \left\{\begin{array}{l}
y_{1}(2)=y_{1}^{-1} \\
y_{2}(2)=y_{2}\left(1 \oplus z y_{1} \oplus y_{1}^{2}\right)
\end{array}\right. \\
& \left\{\begin{array}{l}
x_{1}(3)=x_{1}^{-1} \frac{1+z \hat{y}_{1}+\hat{y}_{1}^{2}}{1 \oplus z y_{1} \oplus y_{1}^{2}} \\
x_{2}(3)=x_{2}^{-1} \frac{1+\hat{y}_{2}+z \hat{y}_{1} \hat{y}_{2}+\hat{y}_{1}^{2} \hat{y}_{2}}{1 \oplus y_{2} \oplus z y_{1} y_{2} \oplus y_{1}^{2} y_{2}}
\end{array}\right. \\
& \left\{\begin{array}{l}
y_{1}(3)=y_{1}^{-1}\left(1 \oplus y_{2} \oplus z y_{1} y_{2} \oplus y_{1}^{2} y_{2}\right) \\
y_{2}(3)=y_{2}^{-1}\left(1 \oplus z y_{1} \oplus y_{1}^{2}\right)^{-1}
\end{array}\right.
\end{aligned}
$$

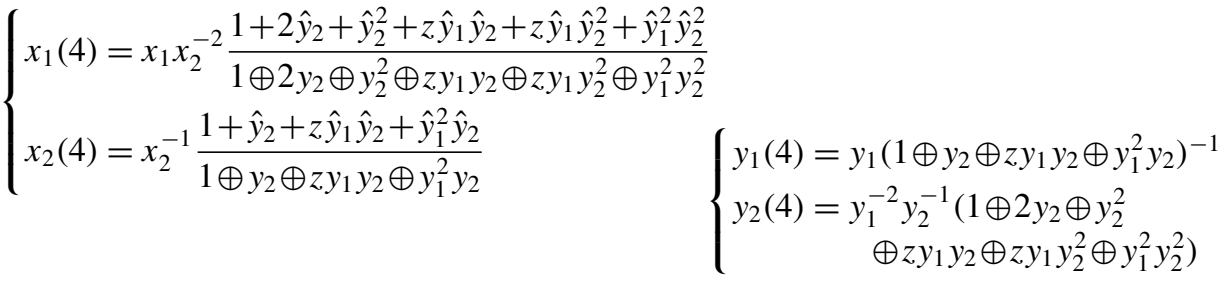

$$
\begin{aligned}
& \left\{\begin{array}{l}
x_{1}(5)=x_{1} x_{2}^{-2} \frac{1+2 \hat{y}_{2}+\hat{y}_{2}^{2}+z \hat{y}_{1} \hat{y}_{2}+z \hat{y}_{1} \hat{y}_{2}^{2}+\hat{y}_{1}^{2} \hat{y}_{2}^{2}}{1 \oplus 2 y_{2} \oplus y_{2}^{2} \oplus z y_{1} y_{2} \oplus z y_{1} y_{2}^{2} \oplus y_{1}^{2} y_{2}^{2}} \\
x_{2}(5)=x_{1} x_{2}^{-1} \frac{1+\hat{y}_{2}}{1 \oplus y_{2}}
\end{array}\right. \\
& \left\{\begin{aligned}
y_{1}(5)= & y_{1}^{-1} y_{2}^{-1}\left(1 \oplus y_{2}\right) \\
y_{2}(5)= & y_{1}^{2} y_{2}\left(1 \oplus 2 y_{2} \oplus y_{2}^{2}\right. \\
& \left.\oplus z y_{1} y_{2} \oplus z y_{1} y_{2}^{2} \oplus y_{1}^{2} y_{2}^{2}\right)^{-1}
\end{aligned}\right. \\
& \left\{\begin{array}{l}
x_{1}(6)=x_{1} \\
x_{2}(6)=x_{1} x_{2}^{-1} \frac{1+\hat{y}_{2}}{1 \oplus y_{2}}
\end{array}\right. \\
& \left\{\begin{array}{l}
y_{1}(6)=y_{1} y_{2}\left(1 \oplus y_{2}\right)^{-1} \\
y_{2}(6)=y_{2}^{-1}
\end{array}\right. \\
& \left\{\begin{array}{l}
x_{1}(7)=x_{1} \\
x_{2}(7)=x_{2}
\end{array}\right. \\
& \left\{\begin{array}{l}
y_{1}(7)=y_{1} \\
y_{2}(7)=y_{2}
\end{array}\right.
\end{aligned}
$$

Table 1. $x$ - and $y$-variables for sequence (2-24).

Definition 3.1. A $(\boldsymbol{d}, \boldsymbol{z})$-cluster pattern with principal coefficients is a $(\boldsymbol{d}, \boldsymbol{z})$-cluster pattern in $\mathbb{P}=\operatorname{Trop}(\boldsymbol{y}, \boldsymbol{z})$ with initial seed $(\boldsymbol{x}, \boldsymbol{y}, B)$, where $\boldsymbol{x}$ and $B$ are arbitrary.

Definition 3.2. Let $\Sigma$ be the $(\boldsymbol{d}, \boldsymbol{z})$-cluster pattern with principal coefficients and initial seed $(\boldsymbol{x}, \boldsymbol{y}, B)$. By the Laurent property in Theorem 2.8, each $x$-variable $x_{i}^{t}$ in $\Sigma$ is expressed as $X_{i}^{t}(\boldsymbol{x}, \boldsymbol{y}, \boldsymbol{z}) \in \mathbb{Z} \mathbb{P}\left[\boldsymbol{x}^{ \pm 1}\right]$ with $\mathbb{P}=\operatorname{Trop}(\boldsymbol{y}, \boldsymbol{z})$. We call them the $X$-functions of $\Sigma$.

For principal coefficients, we actually have the following result, which is stronger than Theorem 2.8 and which is parallel to [Fomin and Zelevinsky 2003, Proposition 11.2; 2007, Proposition 3.6]. 
Proposition 3.3. We have

$$
X_{i}^{t}(\boldsymbol{x}, \boldsymbol{y}, z) \in \mathbb{Z}\left[\boldsymbol{x}^{ \pm 1}, \boldsymbol{y}, \boldsymbol{z}\right] .
$$

Proof. We follow the argument in the proof of [Fomin and Zelevinsky 2003, Proposition 11.2]. Let $p$ be any variable in $\boldsymbol{y}$ or $\boldsymbol{z}$. Let us view $X_{i}^{t}(\boldsymbol{x}, \boldsymbol{y}, \boldsymbol{z})$ as a Laurent polynomial in $p$, say $h(p)$, whose coefficients are Laurent polynomials in the rest of the variables in $\boldsymbol{x}, \boldsymbol{y}$, and $\boldsymbol{z}$. We show that $h(p)$ is a polynomial in $p$ with nonzero constant term having subtraction-free rational expression by induction on the distance between $t$ and $t_{0}$ in $\mathbb{T}_{n}$. The crucial point is that the coefficients $p_{k, s}=z_{k, s} y_{k}^{s} / \bigoplus_{r=0}^{d_{k}} z_{k, r} y_{k}^{r}$ in the mutation (2-6) are normalized as (2-14). Since $\mathbb{P}=\operatorname{Trop}(\boldsymbol{y}, \boldsymbol{z})$, this means that $p_{k, s}\left(s=0, \ldots, d_{r}\right)$ are polynomials in $p$, and there is no common factor in $p$. Thus, the right-hand side of (2-6) is a polynomial in $p$ with nonzero constant term having subtraction-free rational expression by the induction hypothesis and the "trivial lemma" (Lemma 5.2) in [Fomin and Zelevinsky 2003].

Definition 3.4. We denote by $\Sigma$ the $(\boldsymbol{d}, \boldsymbol{z})$-cluster pattern in the universal semifield $\mathbb{Q}_{\mathrm{sf}}(\boldsymbol{y}, \boldsymbol{z})$ with initial seed $(\boldsymbol{x}, \boldsymbol{y}, B)$. Each $y$-variable $y_{i}^{t}$ in $\Sigma$ is expressed as a subtraction-free rational function $Y_{i}^{t}(\boldsymbol{y}, \boldsymbol{z}) \in \mathbb{Q}_{\mathrm{sf}}(\boldsymbol{y}, \boldsymbol{z})$. We call them the $Y$-functions of $\Sigma$.

Due to the universal property of the semifield $\mathbb{Q}_{s f}(\boldsymbol{y}, \boldsymbol{z})$ [Fomin and Zelevinsky 2007, Definition 2.1], the following fact holds.

Lemma 3.5. For any $(\boldsymbol{d}, \boldsymbol{z})$-cluster pattern in $\mathbb{P}$ with the same initial exchange matrix $B$ as above, we have

$$
y_{i}^{t}=\left.Y_{i}^{t}\right|_{\mathbb{P}}(\boldsymbol{y}, \boldsymbol{z}),
$$

where the right-hand side stands for the evaluation of $Y_{i}^{t}(\boldsymbol{y}, \boldsymbol{z})$ in $\mathbb{P}$.

3B. c-vectors, F-polynomials, and g-vectors. Let us extend the notions of $c$ vectors, $F$-polynomials, and $g$-vectors in [Fomin and Zelevinsky 2007] to a $(\boldsymbol{d}, \boldsymbol{z})$ cluster pattern with principal coefficients.

3B.1. $C$-matrices and c-vectors. For a $(\boldsymbol{d}, \boldsymbol{z})$-cluster pattern with principal coefficients, each $y$-variable $y_{i}^{t} \in \operatorname{Trop}(\boldsymbol{y}, \boldsymbol{z})$ is, by definition, a Laurent monomial of $\boldsymbol{y}$ and $z$ with coefficient 1 . The following simple fact was observed in [Iwaki and Nakanishi 2014] in the special case.

Lemma 3.6. Each $y$-variable $y_{i}^{t}$ is actually a Laurent monomial of $\boldsymbol{y}$ with coeffcient 1 .

Proof. This is equivalent to saying that the frozen coefficients $z$ never enter in $y_{i}^{t}$. This is true for the initial $y$-variables. Then, the claim can be shown by induction 
on the distance between $t$ and $t_{0}$ in $\mathbb{T}_{n}$, by inspecting the mutation (2-5) and the definition of the tropical sum (3-1).

Definition 3.7. Let $\Sigma$ be a $(\boldsymbol{d}, \boldsymbol{z})$-cluster pattern with principal coefficients. Let us express each $y$-variable $y_{j}^{t}$ in $\Sigma$ as

$$
y_{j}^{t}=\left.Y_{i}^{t}\right|_{\operatorname{Trop}(\boldsymbol{y}, \boldsymbol{z})}(\boldsymbol{y}, \boldsymbol{z})=\prod_{i=1}^{n} y_{i}^{c_{i j}^{t}} .
$$

The resulting matrices $C^{t}=\left(c_{i j}^{t}\right)_{i, j=1}^{n}$ and their column vectors $c_{j}^{t}=\left(c_{i j}^{t}\right)_{i=1}^{n}$ are called the $C$-matrices and the $c$-vectors of $\Sigma$, respectively.

The following mutation/recurrence formula provides a combinatorial description of $c$-vectors.

Proposition 3.8. The $c$-vectors of a $(\boldsymbol{d}, \boldsymbol{z})$-cluster pattern with principal coefficients satisfy the following recurrence relation for $t \underline{k} t^{\prime}$ :

$$
\begin{aligned}
c_{i j}^{t_{0}} & =\delta_{i j}, \\
c_{i j}^{t^{\prime}} & = \begin{cases}-c_{i k}^{t} & \text { if } j=k, \\
c_{i j}^{t}+c_{i k}^{t}\left[\varepsilon d_{k} b_{k j}^{t}\right]_{+}+\left[-\varepsilon c_{i k}^{t}\right]_{+} d_{k} b_{k j}^{t} & \text { if } j \neq k,\end{cases}
\end{aligned}
$$

where $\varepsilon= \pm 1$ and it is independent of the choice of the sign $\varepsilon$.

Proof. As already remarked in the proof of Lemma 3.6, for a $(\boldsymbol{d}, \boldsymbol{z})$-cluster pattern with principal coefficients, the mutation (2-5) is simplified as

$$
y_{i}^{t^{\prime}}= \begin{cases}y_{k}^{t-1} & \text { if } i=k, \\ y_{i}^{t}\left(y_{k}^{t}\left[\varepsilon b_{k i}^{t}\right]_{+}\right)^{d_{k}}\left(\bigoplus_{s=0}^{d_{k}} y_{k}^{t \varepsilon s}\right)^{-b_{k i}^{t}} & \text { if } i \neq k .\end{cases}
$$

This is equivalent to (3-6) due to the following formula in $\operatorname{Trop}(\boldsymbol{y}, \boldsymbol{z})$ :

$$
\frac{1}{\bigoplus_{s=0}^{d_{k}}\left(\prod_{j=1}^{n} y_{j}^{\varepsilon c_{j k}^{t}}\right)^{s}}=\left(\prod_{j=1}^{n} y_{j}^{\left[-\varepsilon c_{j k}^{t}\right]_{+}}\right)^{d_{k}} .
$$

We observe that the above relation coincides with the one for the $c$-vectors of the ordinary cluster pattern with principal coefficients and initial seed $(\boldsymbol{x}, \boldsymbol{y}, D B)$ in [Fomin and Zelevinsky 2002, Proposition 5.8]. Therefore, we have the following result.

Proposition 3.9. The c-vectors of the $(\boldsymbol{d}, \boldsymbol{z})$-cluster pattern with principal coeffcients and initial seed $(\boldsymbol{x}, \boldsymbol{y}, B)$ coincide with the c-vectors of the ordinary cluster pattern with principal coefficients and initial seed $(\boldsymbol{x}, \boldsymbol{y}, D B)$. 
Alternatively, one can relate these $c$-vectors with the $c$-vectors of the ordinary cluster pattern with principal coefficients and initial seed $(\boldsymbol{x}, \boldsymbol{y}, B D)$ as follows. Let us introduce

$$
\tilde{c}_{i j}^{t}=d_{i}^{-1} c_{i j}^{t} d_{j}
$$

Then, $\tilde{c}_{i j}^{t_{0}}=\delta_{i j}$, and (3-6) is rewritten as

$$
\tilde{c}_{i j}^{t^{\prime}}= \begin{cases}-\tilde{c}_{i k}^{t} & \text { if } j=k \\ \tilde{c}_{i j}^{t}+\tilde{c}_{i k}^{t}\left[\varepsilon b_{k j}^{t} d_{j}\right]_{+}+\left[-\varepsilon \tilde{c}_{i k}^{t}\right]_{+} b_{k j}^{t} d_{j} & \text { if } j \neq k\end{cases}
$$

Therefore, we have the following result.

Proposition 3.10. The $\tilde{c}$-vectors, which are the column vectors in (3-9), of the $(\boldsymbol{d}, \boldsymbol{z})$-cluster pattern with principal coefficients and initial seed $(\boldsymbol{x}, \boldsymbol{y}, B)$ coincide with the $c$-vectors of the ordinary cluster pattern with principal coefficients and initial seed $(\boldsymbol{x}, \boldsymbol{y}, B D)$.

We need this alternative description for the description of the $g$-vectors below.

3B.2. F-polynomials. Thanks to Proposition 3.3, the following definition makes sense.

Definition 3.11. Let $\Sigma$ be a $(\boldsymbol{d}, \boldsymbol{z})$-cluster pattern with principal coefficients. For each $t \in \mathbb{T}_{n}$ and $i=1, \ldots, n$, a polynomial $F_{i}^{t}(\boldsymbol{y}, \boldsymbol{z}) \in \mathbb{Z}[\boldsymbol{y}, \boldsymbol{z}]$ is defined by the specialization of the $X$-function $X_{i}^{t}(\boldsymbol{x}, \boldsymbol{y}, \boldsymbol{z})$ of $\Sigma$ with $x_{1}=\cdots=x_{n}=1$. They are called the $F$-polynomials of $\Sigma$.

The following mutation/recurrence formula provides a combinatorial description of $F$-polynomials.

Proposition 3.12 (cf. [Fomin and Zelevinsky 2007, Proposition 5.1]). The Fpolynomials for a $(\boldsymbol{d}, \boldsymbol{z})$-cluster pattern with principal coefficients satisfy the following recurrence relation for $t \underline{k} t^{\prime}$ :

(3-11) $F_{i}^{t_{0}}=1$,

$$
F_{i}^{t^{\prime}}= \begin{cases}F_{k}^{t-1}\left(\prod_{j=1}^{n} y_{j}^{\left[-\varepsilon c_{j k}^{t}\right]_{+}} F_{j}^{t\left[-\varepsilon b_{j k}^{t}\right]_{+}}\right)^{d_{k}} \sum_{s=0}^{d_{k}} z_{k, s}\left(\prod_{j=1}^{n} y_{j}^{\varepsilon c_{j k}^{t}} F_{j}^{t \varepsilon b_{j k}^{t}}\right)^{s} & \text { if } i=k, \\ F_{i}^{t} & \text { if } i \neq k,\end{cases}
$$

where $\varepsilon= \pm 1$ and it is independent of the choice of the sign $\varepsilon$. 
Proof. By specializing the mutation (2-6) with $\mathbb{P}=\operatorname{Trop}(\boldsymbol{y}, \boldsymbol{z})$, we obtain

$$
X_{i}^{t^{\prime}}= \begin{cases}X_{k}^{t-1}\left(\prod_{j=1}^{n} X_{j}^{t\left[-\varepsilon b_{j k}^{t}\right]_{+}}\right)^{d_{k}} \frac{\sum_{s=0}^{d_{k}} z_{k, s}\left(\prod_{j=1}^{n} y_{j}^{\varepsilon c_{j k}^{t}} X_{j}^{t \varepsilon b_{j k}^{t}}\right)^{s}}{\bigoplus_{s=0}^{d_{k}}\left(\prod_{j=1}^{n} y_{j}^{t} c_{j k}^{t}\right)^{s}} & \text { if } i=k, \\ X_{i}^{t} & \text { if } i \neq k .\end{cases}
$$

Then, specializing it with $x_{1}=\ldots x_{n}=1$, and using (3-8), we obtain (3-12).

3B.3. $G$-matrices and $g$-vectors. Let $\Sigma$ be the $(\boldsymbol{d}, \boldsymbol{z})$-cluster pattern with principal coefficients and initial seed $(\boldsymbol{x}, \boldsymbol{y}, B)$. Let $\mathbb{Z}\left[\boldsymbol{x}^{ \pm 1}, \boldsymbol{y}, \boldsymbol{z}\right]$ be the one in Proposition 3.3. Following [Fomin and Zelevinsky 2007], we introduce a $\mathbb{Z}^{n}$-grading in $\mathbb{Z}\left[\boldsymbol{x}^{ \pm 1}, \boldsymbol{y}, \boldsymbol{z}\right]$ as follows:

$$
\operatorname{deg}\left(x_{i}\right)=\boldsymbol{e}_{i}, \quad \operatorname{deg}\left(y_{i}\right)=-\boldsymbol{b}_{j}, \quad \operatorname{deg}\left(z_{i, r}\right)=0 .
$$

Here, $\boldsymbol{e}_{i}$ is the $i$-th unit vector of $\mathbb{Z}^{n}$, and $\boldsymbol{b}_{j}=\sum_{i=1}^{n} b_{i j} \boldsymbol{e}_{i}$ is the $j$-th column of the initial matrix $B=\left(b_{i j}\right)_{i, j=1}^{n}$. Note that $\operatorname{deg}\left(\hat{y}_{i}\right)=0$ by $(2-7)$.

Proposition 3.13 (cf. [Fomin and Zelevinsky 2007, Proposition 6.1]). The $X$ functions are homogeneous with respect to the $\mathbb{Z}^{n}$-grading.

Proof. We repeat the original argument of Fomin and Zelevinsky, by induction on the distance between $t$ and $t_{0}$ in $\mathbb{T}_{n}$. Using (2-6) and Lemma 3.5 specialized to a $(\boldsymbol{d}, \boldsymbol{z})$-cluster pattern with principal coefficients, we have

$$
X_{i}^{t^{\prime}}= \begin{cases}X_{k}^{t-1}\left(\prod_{j=1}^{n} X_{j}^{t\left[-\varepsilon b_{j k}^{t}\right]_{+}}\right)^{d_{k}} \frac{\left.\sum_{s=0}^{d_{k}} z_{k, s} Y_{k}^{t \varepsilon s}\right|_{\mathcal{F}}(\hat{\boldsymbol{y}}, \boldsymbol{z})}{\left.\bigoplus_{s=0}^{d_{k} z_{k, s} Y_{k}^{t} \varepsilon s}\right|_{\operatorname{Trop}(\boldsymbol{y}, \boldsymbol{z})}(\boldsymbol{y}, \boldsymbol{z})} & \text { if } i=k, \\ X_{i}^{t} & \text { if } i \neq k .\end{cases}
$$

Then, the right-hand side is homogeneous due to the induction hypothesis.

Definition 3.14. Let $\Sigma$ be the $(\boldsymbol{d}, \boldsymbol{z})$-cluster pattern with principal coefficients and initial matrix $(\boldsymbol{x}, \boldsymbol{y}, B)$. Thanks to Proposition 3.13, the degree vector $\operatorname{deg}\left(X_{i}^{t}\right)$ of each $X$-function $X_{i}^{t}$ of $\Sigma$ is defined. Let us express it as

$$
\operatorname{deg}\left(X_{j}^{t}\right)=\sum_{i=1}^{n} g_{i j}^{t} \boldsymbol{e}_{i} .
$$

The resulting matrices $G^{t}=\left(g_{i j}^{t}\right)_{i, j=1}^{n}$ and their column vectors $g_{j}^{t}=\left(g_{i j}^{t}\right)_{i=1}^{n}$ are called the $G$-matrices and the $g$-vectors of $\Sigma$, respectively.

The following mutation/recurrence formula provides a combinatorial description of $g$-vectors. 
Proposition 3.15. The g-vectors of the $(\boldsymbol{d}, \boldsymbol{z})$-cluster pattern with principal coefficients and initial seed $(\boldsymbol{x}, \boldsymbol{y}, B)$ satisfy the following recurrence relation for $t \underline{k} t^{\prime}$ :

$$
\begin{aligned}
& g_{i j}^{t_{0}}=\delta_{i j}, \\
& g_{i j}^{t^{\prime}}= \begin{cases}-g_{i k}^{t}+\sum_{\ell=1}^{n} g_{i \ell}^{t}\left[-\varepsilon b_{\ell k}^{t} d_{k}\right]_{+}-\sum_{\ell=1}^{n} b_{i \ell}\left[-\varepsilon c_{\ell k}^{t} d_{k}\right]_{+} & \text {if } j=k, \\
g_{i j}^{t} & \text { if } j \neq k,\end{cases}
\end{aligned}
$$

where $\varepsilon= \pm 1$ and it is independent of the choice of the sign $\varepsilon$.

Proof. This is obtained by comparing the degrees of both sides of (3-13).

By using the $\tilde{c}$-vectors in (3-9), the relation (3-18) is rewritten as follows.

$$
g_{i j}^{t^{\prime}}= \begin{cases}-g_{i k}^{t}+\sum_{\ell=1}^{n} g_{i \ell}^{t}\left[-\varepsilon b_{\ell k}^{t} d_{k}\right]_{+}-\sum_{\ell=1}^{n} b_{i \ell} d_{\ell}\left[-\varepsilon \tilde{c}_{\ell k}^{t}\right]_{+} & \text {if } j=k, \\ g_{i j}^{t} & \text { if } j \neq k .\end{cases}
$$

Having Proposition 3.10 in mind, we observe that this relation coincides with the one for the $g$-vectors of the ordinary cluster pattern with principal coefficients and initial seed $(\boldsymbol{x}, \boldsymbol{y}, B D)$ in [Fomin and Zelevinsky 2007, Proposition 6.6]. Therefore, we have the following result.

Proposition 3.16. The g-vectors of the $(\boldsymbol{d}, \boldsymbol{z})$-cluster pattern with principal coeffcients and initial seed $(\boldsymbol{x}, \boldsymbol{y}, B)$ coincide with the g-vectors of the ordinary cluster pattern with principal coefficients and initial seed $(\boldsymbol{x}, \boldsymbol{y}, B D)$.

For the sake of completeness, we also present the counterpart of Proposition 3.10. Let us introduce

$$
\tilde{g}_{i j}^{t}=d_{i} g_{i j}^{t} d_{j}^{-1} .
$$

Then, the relation (3-18) is also rewritten as

$$
\tilde{g}_{i j}^{t^{\prime}}= \begin{cases}-\tilde{g}_{i k}^{t}+\sum_{\ell=1}^{n} \tilde{g}_{i \ell}^{t}\left[-\varepsilon d_{\ell} b_{\ell k}^{t}\right]_{+}-\sum_{\ell=1}^{n} d_{i} b_{i \ell}\left[-\varepsilon c_{\ell k}^{t}\right]_{+} & \text {if } j=k, \\ \tilde{g}_{i j}^{t} & \text { if } j \neq k .\end{cases}
$$

Having Proposition 3.9 in mind, we observe that this relation coincides with the one for the $g$-vectors of the ordinary cluster pattern with principal coefficients and initial seed $(\boldsymbol{x}, \boldsymbol{y}, D B)$. Therefore, we have the following result.

Proposition 3.17. The $\tilde{g}$-vectors, which are the column vectors in (3-20), of the $(\boldsymbol{d}, \boldsymbol{z})$-cluster pattern with principal coefficients and initial seed $(\boldsymbol{x}, \boldsymbol{y}, B)$ coincide with the g-vectors of the ordinary cluster pattern with principal coefficients and initial seed $(\boldsymbol{x}, \boldsymbol{y}, D B)$. 
We see a duality between the $c$-vectors and the $g$-vectors in Propositions 3.9, $3.10,3.16$, and 3.17. In particular, the $c$-vectors are associated with the matrix $D B$, while the $g$-vectors are associated with the matrix $B D$. This is somewhat suggested from the beginning in the monomial parts in the relations (2-5) and (2-6).

3B.4. Sign-coherence.

Definition 3.18. Let $\Sigma$ be a $(\boldsymbol{d}, \boldsymbol{z})$-cluster pattern with principal coefficients. A $c$-vector $c_{j}^{t}$ of $\Sigma$ is said to be sign-coherent if it is nonzero and all components are either nonnegative or nonpositive.

Proposition 3.19 (cf. [Fomin and Zelevinsky 2007, Proposition 5.6]). For any $(\boldsymbol{d}, \boldsymbol{z})$-cluster pattern with principal coefficients, the following two conditions are equivalent.

(i) Any F-polynomial $F_{i}^{t}(\boldsymbol{y}, \boldsymbol{z})$ has constant term 1.

(ii) Any c-vector $c_{i}^{t}$ is sign-coherent.

Proof. This is proved by an argument parallel to the one in [Fomin and Zelevinsky 2007, Proposition 5.6] by using the recursion relation (3-12) for the $F$-polynomials. We omit the details.

In the ordinary case it was conjectured in [Fomin and Zelevinsky 2007, Conjecture 5.6] that the sign-coherence holds for any $c$-vector of any cluster pattern with principal coefficients. This was proved by Derksen et al. [2010, Theorem 1.7] when the initial exchange matrix $B$ is skew-symmetric, and very recently it was proved in full generality by Gross et al. [2014, Corollary 5.5]. Since our $c$-vectors are identified with the $c$-vectors of some ordinary cluster pattern with principal coefficients by Proposition 3.9, we obtain the following theorem as a corollary of [Gross et al. 2014, Corollary 5.5].

Theorem 3.20. Any c-vector of any $(\boldsymbol{d}, \boldsymbol{z})$-cluster pattern with principal coefficients is sign-coherent.

As a consequence of the sign-coherence, we also obtain the following duality between the $C$ - and $G$-matrices by applying [Nakanishi and Zelevinsky 2012, Equation (3.11)] (see also [Nakanishi 2012, Proposition 3.2]), which is valid under the sign-coherence property. Recall that for a skew-symmetrizable matrix $B$ the matrix $D B$ is still skew-symmetrizable.

Proposition 3.21 (cf. [Nakanishi and Zelevinsky 2012, Equation (3.11)]). Let $C^{t}$ and $G^{t}$ be the $C$-and $G$-matrices at $t \in \mathbb{T}_{n}$ of any $(\boldsymbol{d}, \boldsymbol{z})$-cluster pattern $\Sigma$ with principal coefficients. Let $R=\left(r_{i} \delta_{i j}\right)_{i, j=1}^{n}$ be a diagonal matrix with positive diagonal entries such that $R D B$ is skew-symmetric. Then

$$
R^{-1} D^{-1}\left(G^{t}\right)^{T} D R C^{t}=I .
$$


Proof. This is obtained by combining [Nakanishi and Zelevinsky 2012, Equation (3.11)] with Propositions 3.9 and 3.17.

3C. Main formulas. Finally, we present the main formulas expressing the $x$ - and $y$-variables of any $(\boldsymbol{d}, \boldsymbol{z})$-cluster pattern $\Sigma$ in any semifield $\mathbb{P}$ in terms of $F$-polynomials, $c$-vectors, and $g$-vectors defined for the same initial exchange matrix of $\Sigma$.

Theorem 3.22 (cf. [Fomin and Zelevinsky 2007, Proposition 3.13]). For any $(\boldsymbol{d}, \boldsymbol{z})$ cluster pattern in $\mathbb{P}$,

$$
y_{i}^{t}=\left.\prod_{j=1}^{n} y_{j}^{c_{j i}^{t}} \prod_{j=1}^{n} F_{j}^{t}\right|_{\mathbb{P}}(\boldsymbol{y}, \boldsymbol{z})^{b_{j i}^{t}} .
$$

Proof. We apply Lemma 3.5 to a $(\boldsymbol{d}, \boldsymbol{z})$-cluster pattern with principal coefficients, and we obtain

$$
\hat{y}_{i}^{t}=Y_{i}^{t}(\hat{\boldsymbol{y}}, z) .
$$

On the other hand, specializing (2-7) to the same $(\boldsymbol{d}, \boldsymbol{z})$-cluster pattern with principal coefficients, we have

$$
\hat{y}_{i}^{t}=\left.Y_{i}^{t}\right|_{\operatorname{Trop}(\boldsymbol{y}, \boldsymbol{z})}(\boldsymbol{y}, \boldsymbol{z}) \prod_{j=1}^{n} X_{j}^{t}(\boldsymbol{x}, \boldsymbol{y}, \boldsymbol{z})^{b_{j i}^{t}}=\prod_{j=1}^{n} y_{j}^{c_{j i}^{t}} \prod_{j=1}^{n} X_{j}^{t}(\boldsymbol{x}, \boldsymbol{y}, \boldsymbol{z})^{b_{j i}^{t}},
$$

where we used (3-4) in the second equality. Thus, we have

$$
Y_{i}^{t}(\hat{\boldsymbol{y}}, \boldsymbol{z})=\prod_{j=1}^{n} y_{j}^{c_{j i}^{t}} \prod_{j=1}^{n} X_{j}^{t}(\boldsymbol{x}, \boldsymbol{y}, \boldsymbol{z})^{b_{j i}^{t}}
$$

Now, we set $x_{1}=\cdots=x_{n}=1$. Then, $\hat{\boldsymbol{y}}=\boldsymbol{y}$, and we obtain

$$
Y_{i}^{t}(\boldsymbol{y}, \boldsymbol{z})=\prod_{j=1}^{n} y_{j}^{c_{j i}^{t}} \prod_{j=1}^{n} F_{j}^{t}(\boldsymbol{y}, \boldsymbol{z})^{b_{j i}^{t}}
$$

Finally, evaluating it in $\mathbb{P}$, we obtain (3-23).

Theorem 3.23 (cf. [Fomin and Zelevinsky 2007, Corollary 6.3]). For any $(\boldsymbol{d}, \boldsymbol{z})$ cluster pattern in $\mathbb{P}$,

$$
x_{i}^{t}=\left(\prod_{j=1}^{n} x_{j}^{g_{j i}^{t}}\right) \frac{\left.F_{i}^{t}\right|_{\mathcal{F}}(\hat{\boldsymbol{y}}, \boldsymbol{z})}{\left.F_{i}^{t}\right|_{\mathbb{P}}(\boldsymbol{y}, \boldsymbol{z})} .
$$

Proof. First, we obtain the following equality in exactly the same way as [Fomin and Zelevinsky 2007, Theorem 3.7], and we skip its derivation: 
$(3-29)$

$$
x_{i}^{t}=\frac{\left.X_{i}^{t}\right|_{\mathcal{F}}(\boldsymbol{x}, \boldsymbol{y}, \boldsymbol{z})}{\left.F_{i}^{t}\right|_{\mathbb{P}}(\boldsymbol{y}, \boldsymbol{z})} .
$$

On the other hand, by the definition of the $g$-vectors, we have (3-30)

$$
X_{i}^{t}\left(\ldots, \gamma_{i} x_{i}, \ldots ; \ldots, \prod_{j=1}^{n} \gamma_{k}^{-b_{k i}} y_{i}, \ldots ; \ldots, z_{i, r}, \ldots\right)=\left(\prod_{j=1} \gamma_{j}^{g_{j i}^{t}}\right) X_{i}^{t}(\boldsymbol{x}, \boldsymbol{y}, \boldsymbol{z}) .
$$

By setting $\gamma_{i}=x_{i}^{-1}$, we have

$$
F_{i}^{t}(\hat{\boldsymbol{y}}, \boldsymbol{z})=\left(\prod_{j=1} x_{j}^{-g_{j i}^{t}}\right) X_{i}^{t}(\boldsymbol{x}, \boldsymbol{y}, \boldsymbol{z}) .
$$

Combining it with (3-29), we obtain (3-28).

3D. Example. Let us consider the example in Section $2 \mathrm{C}$ again. From the data in Table 1, one can read off the following data for the $C$-matrix $C(t)$, the $G$-matrix $G(t)$, and the $F$-polynomials $F_{i}(t)$ for the seed $\Sigma(t)$ with principal coefficients therein.

$$
\begin{array}{lll}
C(1)=\left(\begin{array}{ll}
1 & 0 \\
0 & 1
\end{array}\right), & G(1)=\left(\begin{array}{ll}
1 & 0 \\
0 & 1
\end{array}\right), & \left\{\begin{array}{l}
F_{1}(1)=1, \\
F_{2}(1)=1,
\end{array}\right. \\
C(2)=\left(\begin{array}{rr}
-1 & 0 \\
0 & 1
\end{array}\right), & G(2)=\left(\begin{array}{rr}
-1 & 0 \\
0 & 1
\end{array}\right), & \left\{\begin{array}{l}
F_{1}(2)=1+z y_{1}+y_{1}^{2}, \\
F_{2}(2)=1,
\end{array}\right. \\
C(3)=\left(\begin{array}{rr}
-1 & 0 \\
0 & -1
\end{array}\right), \quad G(3)=\left(\begin{array}{rr}
-1 & 0 \\
0 & -1
\end{array}\right), & \left\{\begin{array}{l}
F_{1}(3)=1+z y_{1}+y_{1}^{2}, \\
F_{2}(3)=1+y_{2}+z y_{1} y_{2}+y_{1}^{2} y_{2},
\end{array}\right. \\
C(4)=\left(\begin{array}{rr}
1 & -2 \\
0 & -1
\end{array}\right), & G(4)=\left(\begin{array}{rr}
1 & 0 \\
-2 & -1
\end{array}\right), & \left\{\begin{array}{l}
F_{1}(4)=1+2 y_{2}+y_{2}^{2} \\
F_{2}(4)=1+y_{1} y_{2}+z y_{1} y_{2}^{2}+y_{1}^{2} y_{2}^{2},
\end{array}\right. \\
C(5)=\left(\begin{array}{rr}
-1 & 2 \\
-1 & 1
\end{array}\right), & G(5)=\left(\begin{array}{rr}
1 & 1 \\
-2 & -1
\end{array}\right), & \left\{\begin{array}{l}
F_{1}(5)=1+2 y_{2}+y_{2}^{2} y_{2}, \\
F_{2}(5)=1+y_{1} y_{2}+z y_{1} y_{2}^{2}+y_{1}^{2} y_{2}^{2},
\end{array}\right. \\
C(6)=\left(\begin{array}{ll}
1 & 0 \\
1 & -1
\end{array}\right), & G(6)=\left(\begin{array}{ll}
1 & 1 \\
0 & -1
\end{array}\right), & \left\{\begin{array}{l}
F_{1}(6)=1, \\
F_{2}(6)=1+y_{2},
\end{array}\right. \\
C(7)=\left(\begin{array}{ll}
1 & 0 \\
0 & 1
\end{array}\right), & G(7)=\left(\begin{array}{ll}
1 & 0 \\
0 & 1
\end{array}\right), & \left\{\begin{array}{l}
F_{1}(7)=1, \\
F_{2}(7)=1 .
\end{array}\right.
\end{array}
$$

\section{Acknowledgements}

We thank Anne-Sophie Gleitz, Kohei Iwaki, and Michael Shapiro for useful discussions and communications. 


\section{References}

[Chekhov and Shapiro 2014] L. Chekhov and M. Shapiro, "Teichmüller spaces of Riemann surfaces with orbifold points of arbitrary order and cluster variables", Int. Math. Res. Not. 2014:10 (2014), 2746-2772. MR 3214284 Zbl 1301.30042

[Derksen et al. 2010] H. Derksen, J. Weyman, and A. Zelevinsky, "Quivers with potentials and their representations, II: Applications to cluster algebras", J. Amer. Math. Soc. 23:3 (2010), 749-790. MR 2012c:16044 Zbl 1208.16017

[Fomin and Zelevinsky 2002] S. Fomin and A. Zelevinsky, "Cluster algebras, I: Foundations", J. Amer. Math. Soc. 15:2 (2002), 497-529. MR 2003f:16050 Zbl 1021.16017

[Fomin and Zelevinsky 2003] S. Fomin and A. Zelevinsky, "Cluster algebras, II: Finite type classification", Invent. Math. 154:1 (2003), 63-121. MR 2004m:17011 Zbl 1054.17024

[Fomin and Zelevinsky 2007] S. Fomin and A. Zelevinsky, "Cluster algebras, IV: Coefficients", Compos. Math. 143:1 (2007), 112-164. MR 2008d:16049 Zbl 1127.16023

[Gekhtman et al. 2005] M. Gekhtman, M. Shapiro, and A. Vainshtein, "Cluster algebras and WeilPetersson forms”, Duke Math. J. 127:2 (2005), 291-311. Correction at 139:2 (2007), 407-409. MR 2006d:53103 Zbl 1079.53124

[Gleitz 2014] A.-S. Gleitz, "Quantum affine algebras at roots of unity and generalised cluster algebras", preprint, 2014. arXiv 1410.2446

[Gross et al. 2014] M. Gross, P. Hacking, S. Keel, and M. Kontsevich, "Canonical bases for cluster algebras", preprint, 2014. arXiv 1411.1394

[Iwaki and Nakanishi 2014] K. Iwaki and T. Nakanishi, "Exact WKB analysis and cluster algebras, II: Simple poles, orbifold points, and generalized cluster algebras", preprint, 2014. arXiv 1409.4641

[Nakanishi 2012] T. Nakanishi, "Tropicalization method in cluster algebras", pp. 95-115 in Tropical geometry and integrable systems (Glasgow, 2011), edited by C. Athorne et al., Contemp. Math. 580, Amer. Math. Soc., Providence, RI, 2012. MR 2985390

[Nakanishi and Zelevinsky 2012] T. Nakanishi and A. Zelevinsky, "On tropical dualities in cluster algebras", pp. 217-226 in Algebraic groups and quantum groups (Nagoya, 2010), edited by S. Ariki et al., Contemp. Math. 565, Amer. Math. Soc., Providence, RI, 2012. MR 2932428 Zbl 06296873

[Plamondon 2011] P.-G. Plamondon, "Cluster algebras via cluster categories with infinite-dimensional morphism spaces”, Compos. Math. 147:6 (2011), 1921-1954. MR 2862067 Zbl 1244.13017

[Rupel 2013] D. Rupel, “Greedy bases in rank 2 generalized cluster algebras”, preprint, 2013. arXiv 1309.2567

Received November 27, 2014. Revised January 21, 2015.

TOMOKI NAKANISHI

GRADUATE SCHOOL OF MATHEMATICS

NAGOYA UNIVERISTY

CHIKUSA-KU

NAGOYA 464-8602

JAPAN

nakanisi@math.nagoya-u.ac.jp 


\title{
PACIFIC JOURNAL OF MATHEMATICS
}

\author{
msp.org/pjm
}

Founded in 1951 by E. F. Beckenbach (1906-1982) and F. Wolf (1904-1989)

\section{EDITORS}

Don Blasius (Managing Editor)

Department of Mathematics

University of California

Los Angeles, CA 90095-1555

blasius@math.ucla.edu

\author{
Paul Balmer \\ Department of Mathematics \\ University of California \\ Los Angeles, CA 90095-1555 \\ balmer@math.ucla.edu \\ Robert Finn \\ Department of Mathematics \\ Stanford University \\ Stanford, CA 94305-2125 \\ finn@math.stanford.edu \\ Sorin Popa \\ Department of Mathematics \\ University of California \\ Los Angeles, CA 90095-1555 \\ popa@math.ucla.edu
}

\author{
Vyjayanthi Chari \\ Department of Mathematics \\ University of California \\ Riverside, CA 92521-0135 \\ chari@math.ucr.edu \\ Kefeng Liu \\ Department of Mathematics \\ University of California \\ Los Angeles, CA 90095-1555 \\ liu@math.ucla.edu \\ Jie Qing \\ Department of Mathematics \\ University of California \\ Santa Cruz, CA 95064 \\ qing@ cats.ucsc.edu
}

\section{PRODUCTION}

Silvio Levy, Scientific Editor, production@msp.org

\section{SUPPORTING INSTITUTIONS}

ACADEMIA SINICA, TAIPEI

CALIFORNIA INST. OF TECHNOLOGY

INST. DE MATEMÁTICA PURA E APLICADA

KEIO UNIVERSITY

MATH. SCIENCES RESEARCH INSTITUTE

NEW MEXICO STATE UNIV.

OREGON STATE UNIV.

\author{
STANFORD UNIVERSITY \\ UNIV. OF BRITISH COLUMBIA \\ UNIV. OF CALIFORNIA, BERKELEY \\ UNIV. OF CALIFORNIA, DAVIS \\ UNIV. OF CALIFORNIA, LOS ANGELES \\ UNIV. OF CALIFORNIA, RIVERSIDE \\ UNIV. OF CALIFORNIA, SAN DIEGO \\ UNIV. OF CALIF., SANTA BARBARA
}

\author{
Daryl Cooper \\ Department of Mathematics \\ University of California \\ Santa Barbara, CA 93106-3080 \\ cooper@math.ucsb.edu \\ Jiang-Hua Lu \\ Department of Mathematics \\ The University of Hong Kong \\ Pokfulam Rd., Hong Kong \\ jhlu@maths.hku.hk \\ Paul Yang \\ Department of Mathematics \\ Princeton University \\ Princeton NJ 08544-1000 \\ yang@math.princeton.edu
}

These supporting institutions contribute to the cost of publication of this Journal, but they are not owners or publishers and have no responsibility for its contents or policies.

See inside back cover or msp.org/pjm for submission instructions.

The subscription price for 2015 is US \$420/year for the electronic version, and \$570/year for print and electronic.

Subscriptions, requests for back issues and changes of subscribers address should be sent to Pacific Journal of Mathematics, P.O. Box 4163, Berkeley, CA 94704-0163, U.S.A. The Pacific Journal of Mathematics is indexed by Mathematical Reviews, Zentralblatt MATH, PASCAL CNRS Index, Referativnyi Zhurnal, Current Mathematical Publications and Web of Knowledge (Science Citation Index).

The Pacific Journal of Mathematics (ISSN 0030-8730) at the University of California, c/o Department of Mathematics, 798 Evans Hall \#3840, Berkeley, CA 94720-3840, is published twelve times a year. Periodical rate postage paid at Berkeley, CA 94704, and additional mailing offices. POSTMASTER: send address changes to Pacific Journal of Mathematics, P.O. Box 4163, Berkeley, CA 94704-0163.

PJM peer review and production are managed by EditFLOW ${ }^{\circledR}$ from Mathematical Sciences Publishers.

\section{PUBLISHED BY}

\section{mathematical sciences publishers \\ nonprofit scientific publishing}

http://msp.org/

(C) 2015 Mathematical Sciences Publishers 


\section{PACIFIC JOURNAL OF MATHEMATICS}

Volume $277 \quad$ No. $1 \quad$ September 2015

Real positivity and approximate identities in Banach algebras

DAVID P. BLECHER and NARUTAKA OZAWA

On shrinking gradient Ricci solitons with nonnegative sectional curvature 61

Mingliang CAI

From quasimodes to resonances: exponentially decaying perturbations

ORAN GANNOT

A general simple relative trace formula

JAYCE R. GETZ and HEEKYOUNG HAHN

Chern-Simons functions on toric Calabi-Yau threefolds and

Donaldson-Thomas theory

\section{ZHENG HUA}

On the flag curvature of a class of Finsler metrics produced by the navigation problem

LiBING HUANG and XIAOHUAN MO

Angular distribution of diameters for spheres and rays for planes

NOBUHIRO INNAMI and YUYA UNEME

A note on an $L^{p}$-Brunn-Minkowski inequality for convex measures in the 187 unconditional case

ARNAUD MARSIGLIETTI

Structure of seeds in generalized cluster algebras

TOMOKI NAKANISHI

Inequalities of Alexandrov-Fenchel type for convex hypersurfaces in hyperbolic space and in the sphere

Yong WeI and ChangWei XiONG

Upper bounds of root discriminant lower bounds 